\title{
Comparison of Acoustic Emission Characteristics for C/SiC Composite Component Under Combination of Heating and Mechanical Loading
}

\author{
Zhi-Yong Tan $^{1} \cdot$ Chang-Wan Min ${ }^{1} \cdot$ Hong-Wei $\mathrm{Wu}^{1} \cdot$ Yu-Hai Qian ${ }^{2} \cdot$ Mei-Shuan Li $^{2}$
}

Received: 14 September 2016/Revised: 27 October 2016/Published online: 24 March 2017

(C) The Chinese Society for Metals and Springer-Verlag Berlin Heidelberg 2017

\begin{abstract}
The acoustic emission (AE) characteristics of $\mathrm{C} / \mathrm{SiC}$ composite component under various conditions were compared, with the purpose of identifying the possible damage and failure mechanism. During the process of the single mechanical loading, the highest amplitude of the AE signal was less than $85 \mathrm{~dB}$ and the main damage forms of matrix cracking and interface debonding were involved. For the heating process, high-energy AE signals with an amplitude more than $85 \mathrm{~dB}$ were detected and fiber fracture mechanism was determined as well due to the thermal stress caused by the mismatch of the thermal expansion coefficient between the reinforced fiber and matrix. During the combination process of the heating and mechanical loading, it was concluded that the degree of damage was much severer than the simple superposition of damage produced by the individual mechanical loading and the individual heating process.
\end{abstract}

KEY WORDS: C/SiC composite; Damage and failure; Heating; Mechanical loading; Acoustic emission

\section{Introduction}

With the rapid development of aircrafts, especially the continuous pursue of hypersonic velocity, thermal protection materials are being exposed in harsh environments and therefore facing severe technical challenge. Due to their sharper airframe and wider flight envelop, the newly developed components of hypersonic flight vehicles will encounter extremely harsh environment caused by both strong aerodynamic friction heating effect and inevitable mechanical loadings during their service

Available online at http://link.springer.com/journal/40195.

Yu-Hai Qian

yhqian@imr.ac.cn

1 Science and Technology on Space Physics Laboratory, Beijing Institute of Nearspace Vehicle's Systems Engineering, Beijing 100076, China

2 Shenyang National Laboratory for Materials Science, Institute of Metal Research, Chinse Academy of Sciences, Shenyang 110016, China process, and thus more advanced thermal structural and protection materials are urgently needed to improve their service safety. Recently, carbon fiber reinforced composites materials, such as carbon fiber reinforced $\mathrm{SiC}(\mathrm{C} / \mathrm{SiC})$ and carbon fiber reinforced $\mathrm{C}(\mathrm{C} / \mathrm{C})$, have drawn an extensive amount of attentions because of their remarkable combination of various unique properties, such as low density, light weight, high melting point, excellent chemical stability and good thermal shock resistance.

To evaluate the potential application of a new material as ultra-high temperature thermal structural and protection material, the investigation and understanding of its failure behavior in service condition is significantly vital. Unfortunately, it is difficult to in situ identify and determine failure or damage modes of the structural component under its real service condition. Simulating the real service condition in laboratory provides an effective way to understand and evaluate the real application performance of composite material, especially under the complex conditions of the combination of heating process and mechanical loading. However, limited by the current measuring methods and measuring tools, it is still very difficult to 
obtain direct or in situ failure information for composite components under such extremely harsh environment.

Because of good accuracy and sensitivity, especially for its in situ and real-time examination ability, acoustic emission (AE) is becoming a suitable technique for the detection of a wide range of failure processes for structural components of various materials [1-7]. According to the intrinsic parameters or characteristics of AE signal acquired by AE test process, such as amplitude, energy, accumulated counts, rise time, duration time and peak frequency, the failure or damage process of materials under various conditions can be revealed [8]. So it is practical to evaluate the application performance of structural components by the AE test technique.

In the present paper, $\mathrm{AE}$ characteristics for $\mathrm{C} / \mathrm{SiC}$ composite component under the conditions of individual heating, individual mechanical loading and the combination of both them were compared and analyzed. Based on the obtained AE signals, the possible damage mechanism and damage process during the test were identified and determined.

\section{Experimental Process}

\subsection{Test Condition}

The wedge-shaped test specimen was $\mathrm{C}$ fiber reinforced $\mathrm{SiC}(\mathrm{C} / \mathrm{SiC})$ composite component. The dimension of the specimen was $676 \mathrm{~mm}$ in length and $546 \mathrm{~mm}$ in width. The thickness for the thick edge was $95 \mathrm{~mm}$, and the thin edge was $44 \mathrm{~mm}$. The AE signals were acquired during the processes of the single heating, the single mechanical loading and the combination of both them. The heating process was carried out by vacuum quartz tubes with inside electrical resistance element, and the available maximum temperature was $1200{ }^{\circ} \mathrm{C}$. The applied mechanical loading was torque, and its maximum value was $2000 \mathrm{~N} \mathrm{~m}$.

Table 1 Change of the heating temperature and the mechanical loading during the combination of them

\begin{tabular}{lcll}
\hline Time (s) & Mechanical loading (N m) & Time (s) & Heating \\
\hline 300 & 0 & 340 & Begin to heat \\
330 & 1000 & 470 & up to $1200{ }^{\circ} \mathrm{C}$ \\
480 & 1000 & & \\
500 & 2000 & & \\
593.6 & 2000 & 593.6 & Stop to heat \\
599 & 2100 & & \\
604 & 2100 & & \\
614 & 0 & & \\
\hline
\end{tabular}

Table 1 shows the change of the heating temperature obtained and the applied mechanical loading during the combination process of both.

\subsection{AE Test}

The acoustic signal acquisitions were carried out by using a PAC (Physical Acoustics Corporation) acoustic transducer. The entire signals were then acquired and recorded by this transducer. An AE test system with the mode of AE USB by PAC was used to manage the acquisition. Figure 1 presents the schematic diagram for the AE monitoring/acquisition system. In order to avoid the strong destructive effect caused by high temperature during heating process on the AE transducer, a thin Pt wire with length about $1 \mathrm{~m}$ and diameter of $1 \mathrm{~mm}$ was used as the wave guide to transmit the acoustic signals from the tested component. One end of the Pt wire was connected with a ceramic screw located on the bottom of the test component, and the other end was connected with a cone of stainless steel. The top cone plane was coupled with PAC R6 $\alpha$ type resonant transducer $(35-100 \mathrm{kHz}$ acquisition range) by coupling agent.

The signals acquired were amplified by a preamplifier with $40 \mathrm{~dB}$ gain. The parameters defining the signal acquisition were set as follows: The peak definition time (PDT) was $50 \mu \mathrm{s}$, the hit definition time (HDT) was $150 \mu \mathrm{s}$, and the hit lock time (HLT) was $300 \mu \mathrm{s}$. The amplitude distribution covers the range of $0-100 \mathrm{~dB}(0 \mathrm{~dB}$ corresponds to $1 \mathrm{uV}$ at the transducer). The threshold setting of $40 \mathrm{~dB}$ was selected so that no AE noise activity was involved during the test process.

\section{Experimental Results}

\subsection{Single Mechanical Loading}

Figure 2 represents the $\mathrm{AE}$ signals acquired during the process of single mechanical loading. Some main AE

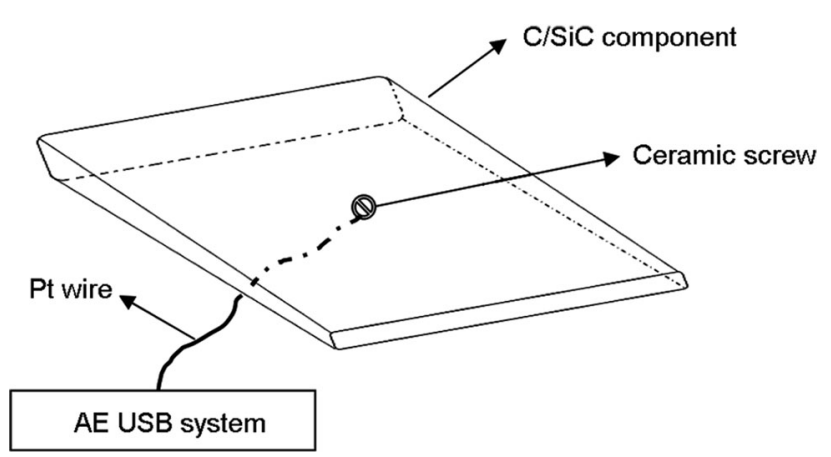

Fig. 1 Schematic diagram for the AE test system 

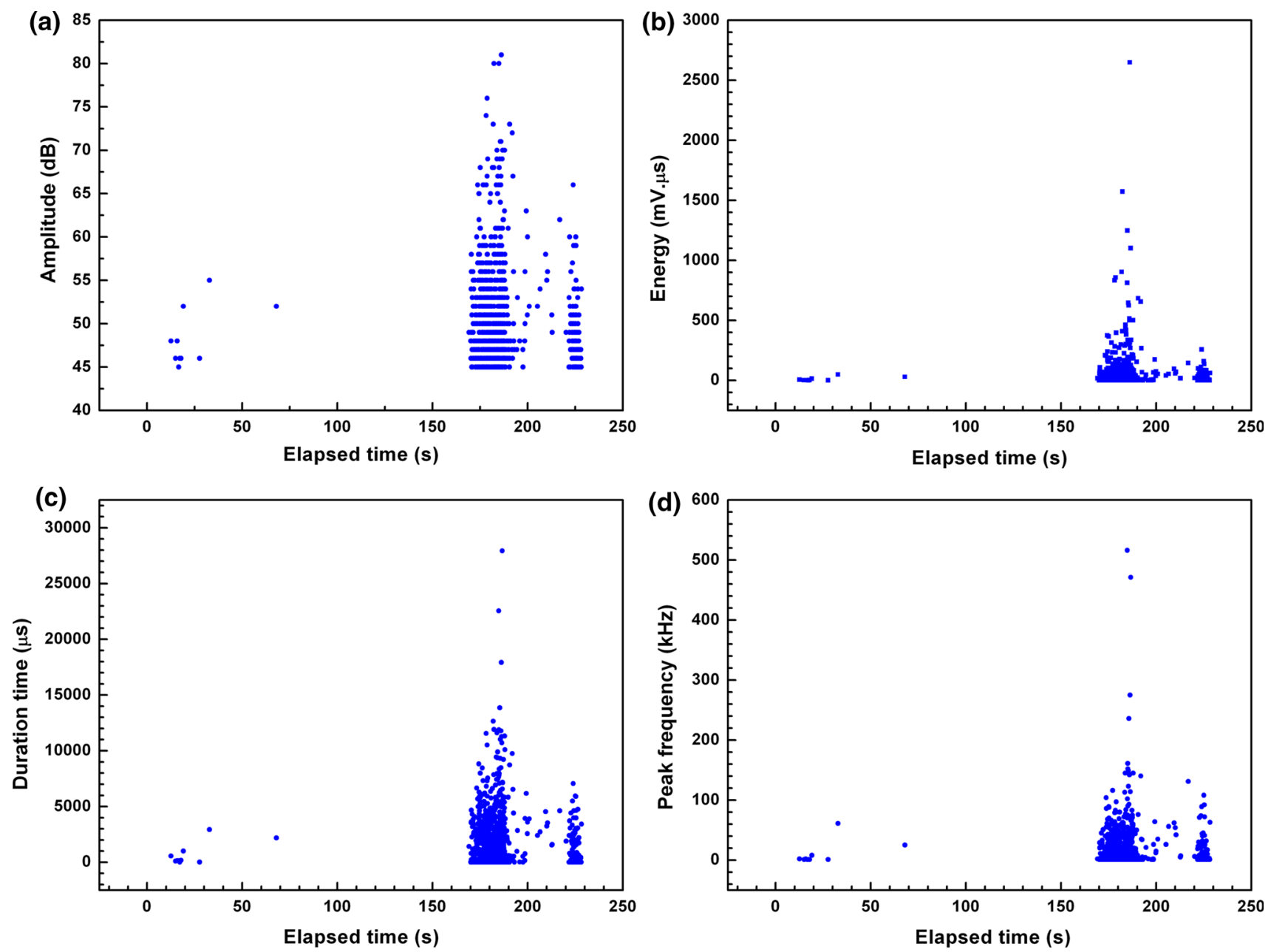

Fig. 2 AE signals during single mechanical loading process: a amplitude distribution, b energy distribution, c duration times distribution, $\mathbf{d}$ peak frequency distribution

parameters including amplitude, energy, duration time and peak frequency are involved, as shown in Fig. 2a, d. From Fig. 2, the whole AE signals exhibit two different phases. The first one belonged to the time period of 168-192 s, which corresponded to the application of maximum torque approximately $2000 \mathrm{~N} \mathrm{~m}$. The second one belonged to the time period of $220-228 \mathrm{~s}$, which corresponded to the applied mechanical torque approximately $1000 \mathrm{~N} \mathrm{~m}$. The torque $1000 \mathrm{~N} \mathrm{~m}$ was applied after the removal of the $2000 \mathrm{~N} \mathrm{~m}$, with the purpose of verifying the reliability of mechanical loading. The initial signals produced from approximately 12-67 s were caused by a strong impact effect during the application of a torque loading.

From Fig. 2, it can be seen that the maximum amplitude of $\mathrm{AE}$ signals is $81 \mathrm{~dB}$, the corresponding maximum energy is $2648 \mathrm{mV}$, the maximum duration time is $27,932 \mu \mathrm{s}$, and the corresponding maximum peak frequency is $516 \mathrm{kHz}$. It should be noted that the $\mathrm{AE}$ parameters acquired during the second phase were significantly lower than those acquired during the first phase.

\subsection{Single Heating Process}

Figure 3 gives the AE signals acquired during the single heating process. According to Fig. 3, after heating for $46 \mathrm{~s}$, the AE signals occurred. Unlike the individual mechanical process, a lot of AE signals appeared during the single heating process (see Fig. 3). Although dense amplitude signals were exhibited, the energy and duration time were very low for most of them. The AE signals with higher energy were focused on mainly two time regions, i.e., 121-164 s and 250-262 s. Meanwhile, at these two time intervals, the corresponding duration time and corresponding peak frequency were high as well.

\subsection{Combination of Heating and Mechanical Loading}

Figure 4 shows the AE signals acquired during the combination of heating and mechanical loading. Like the case of single mechanical loading process, during the initial 

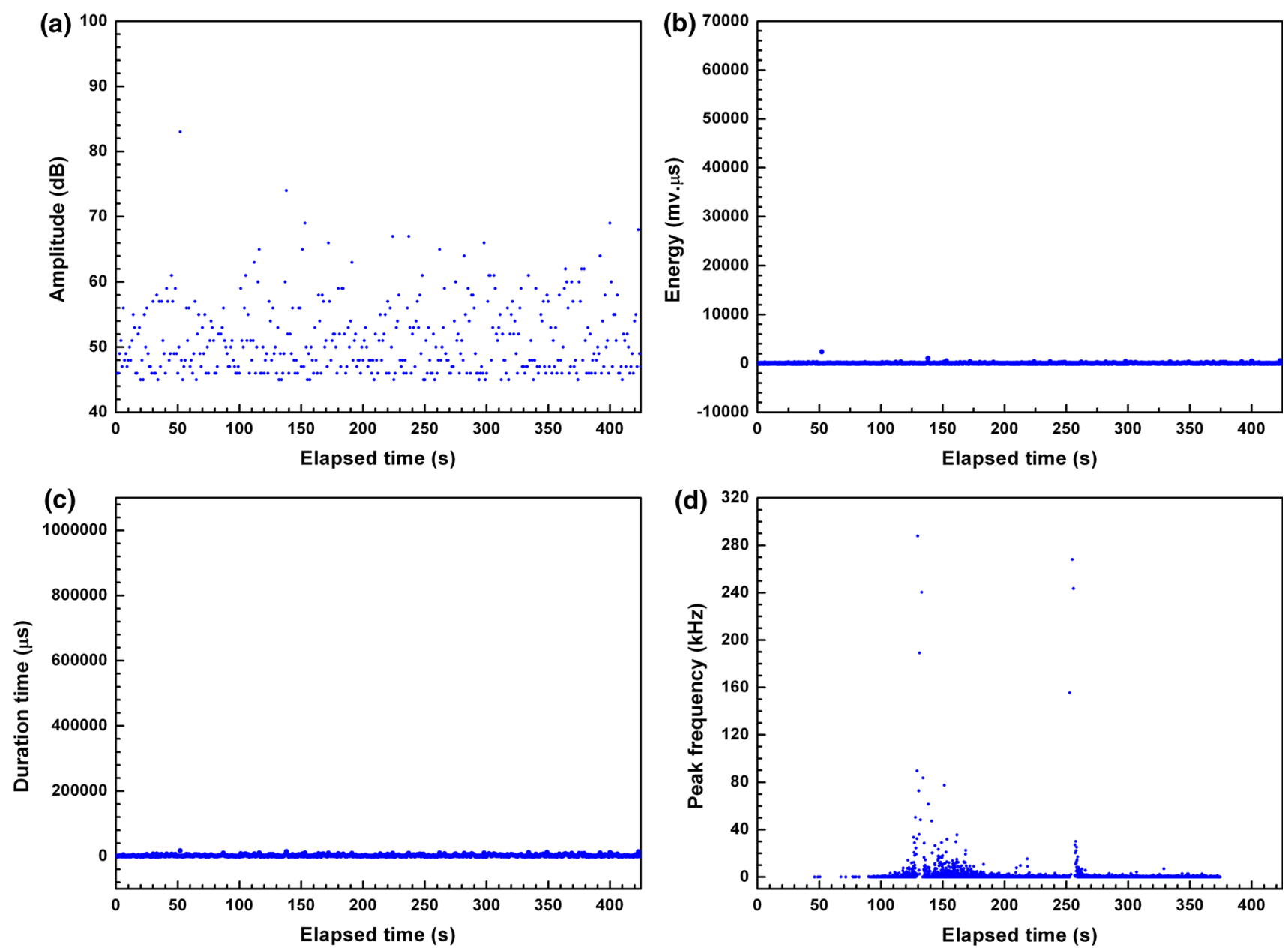

Fig. 3 AE signals during single heating process: a amplitude distribution, $\mathbf{b}$ energy distribution, $\mathbf{c}$ duration times distribution, $\mathbf{d}$ peak frequency distribution

stage, there were some AE signals with much lower amplitude, negligible energy, much lower duration and peak frequency. They may be caused by the impact of initial application of torque mechanical loading. It should be noted that both the shape and distribution of the amplitude were much similar to the one during the singe heating process, but it was much denser. And what's more, the AE signals with higher energy and longer duration time mainly were focused on the time periods of 310-380 s and 455-465 s. Most of the AE signals with relatively low energy and low amplitude appeared at other time periods. From Fig. 4d, it is apparent that the occurrence of high peak frequency is in accordance with the AE signals with high energy and high duration time.

Figure 5 presents the scanning electron microscopy (SEM) micrographs of surface morphology of the failure regions during the combination of both heating and mechanical loading. It is obvious that the modes of damage and failure include crack of matrix and fracture of fiber (Fig. 5a), the interface damage between the matrix and reinforced fibers, i.e., pull-out of fiber (Fig. 5b, c) gives the local magnification view of fracture surface of fibers as well. It could be observed that fracture surfaces were covered with some white ash, resulted from the oxidation or combustion of fibers during the heating process.

\section{Discussion}

The occurrence of AE signals is a result of rapid release of strain energy saved in materials, which is then transmitted in the form of acoustic wave through the body of materials. Commonly, the AE characteristic for fracture behavior of brittle materials exhibits low amplitude and high frequency. On the contrary, the AE characteristic of ductile materials exhibits low frequency and high amplitude. The frequency range of the AE signal depends on the release rate of the strain energy. The more rapid the release rate, the higher the frequency. 

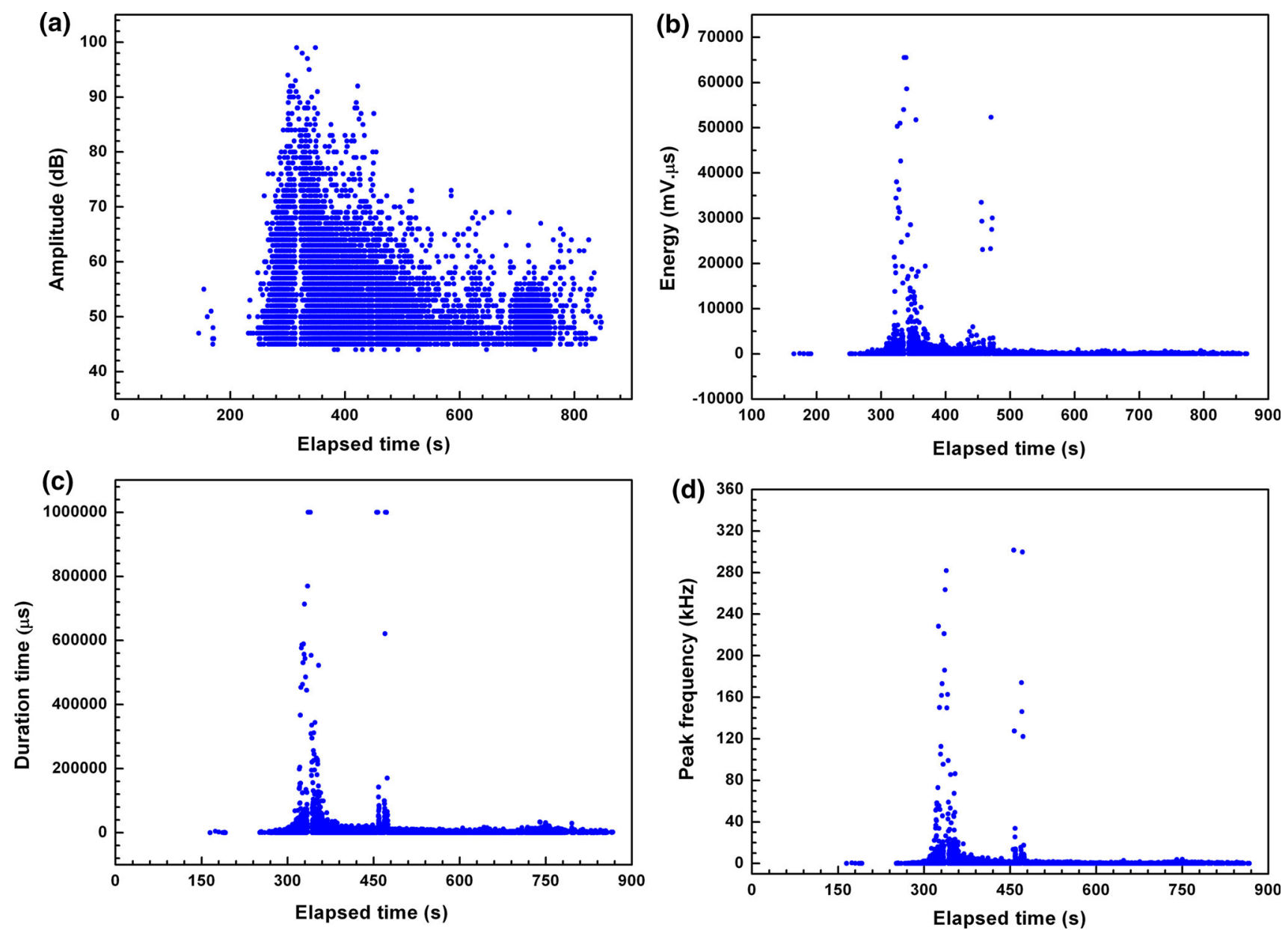

Fig. 4 AE signals during the combination of both heating and mechanical loading: a amplitude distribution, $\mathbf{b}$ energy distribution, $\mathbf{c}$ duration times distribution, $\mathbf{d}$ peak frequency distribution
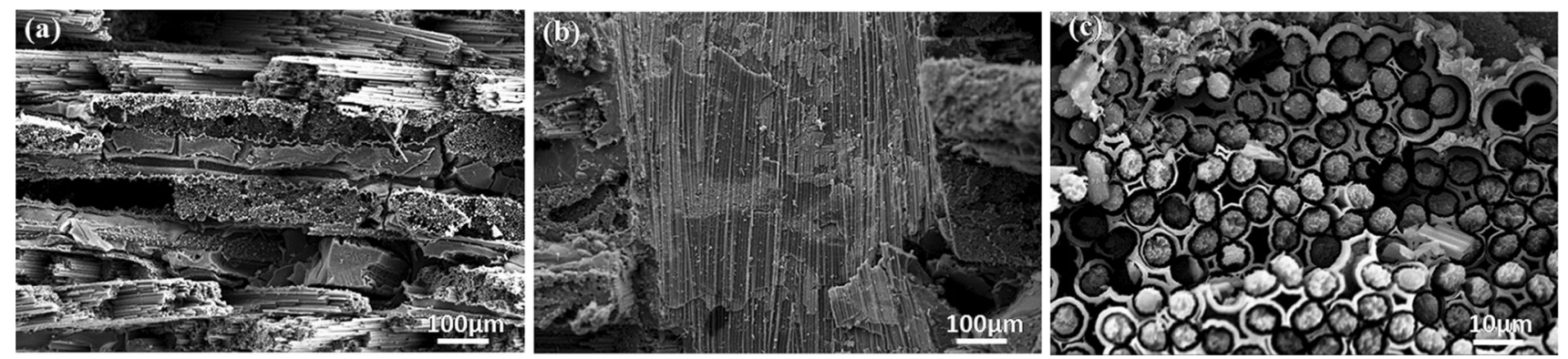

Fig. 5 SEM micrographs of surface morphology of the failure regions during the combination of both heating and mechanical loading: a crack of matrix and fracture of fiber, $\mathbf{b}$ finger-print of pull-out of fiber, $\mathbf{c}$ magnification of fracture of fibers

Commonly, the damage or failure process of materials involves the initiation and expansion of initial microcracks, the expansion of macro-cracks and the latest fracture stage. Each of them gives rise to the occurrence of $\mathrm{AE}$ signals and corresponds to different characteristic of $\mathrm{AE}$ signals. Usually, there are three main well-defined damage or fracture modes of composites, i.e., matrix cracking, debonding between fiber and matrix (including interface damage between the fiber and matrix and fiber pull-out) and fiber failure [9]. For fiber reinforced composites, in the stage of crack initiation and initial expansion phase, the events of $\mathrm{AE}$ are relatively less, the energy is relatively low, and the duration time is short. With the rapid and continuous expansion of cracks, the degree of damage increases, and the number of $\mathrm{AE}$ events and duration time increases as well. Thus, as a typical ceramic matrix 
composite, the damage mechanism of $\mathrm{C}$ fiber reinforced $\mathrm{SiC}(\mathrm{C} / \mathrm{SiC})$ should involve the processes of the initiation and expansion of micro-cracks of $\mathrm{SiC}$ matrix, the de-adhesion of interface between the reinforced $\mathrm{C}$ fiber and $\mathrm{SiC}$ matrix, the fracture and pull-out of the reinforced $\mathrm{C}$ fibers. Due to the unique characteristics of $\mathrm{AE}$ response during various damage phases, it is feasible to describe and determine the types of damage and failure by the comprehensive characteristics of $\mathrm{AE}$ signals acquired.

Generally, the occurrence of $\mathrm{AE}$ events is associated with an increased level of damage in the material. From the literature [10-12], it is known that the AE signals generated are related to the type of fracture mechanism. It is revealed that the lower $\mathrm{AE}$ amplitude distribution characterizes matrix cracking, which is reported and confirmed in various composite systems, such as carbon/epoxy, glass/ polypropylene and glass/polyamide [13-15]. For example, the energy content of the AE signals for glass/polypropylene composite measured by Barre and Benzeggagh [12] revealed that the $\mathrm{AE}$ amplitude from 40 to $55 \mathrm{~dB}$ corresponded to matrix cracking, $60-65 \mathrm{~dB}$ to debonding, $65-85 \mathrm{~dB}$ to glass fiber pull-out and $85-95 \mathrm{~dB}$ to fiber fracture. On the contrary, even if a clear separation of the various damage sources cannot be performed in all cases, the fiber breakage mode showed the highest amplitude [16]. In particular, a time-domain analysis revealed that matrix cracking, in all cases, produces lower amplitude signals than fiber breakage [17, 18]. The frequency information of AE signals also provides an important clue for the damage process of the materials. In fact, acoustic signals of fiber failure are of very small amplitude with respect to the mechanical and electrical noise, but generally, they are characterized by higher frequencies [19]. The frequency analysis of $\mathrm{AE}$ signals for both composites of graphite/epoxy composites and glass/polyester [8,9] suggested that the magnitude of frequency increased in the following order: the matrix cracking $<$ the fiber debonding and pull-out $<$ the fiber breakage. And what's more, fiber breakage exhibited shorter source rise time than debonding [19].

According to Fig. 2, the occurrence of the AE signals is dependent on the level of applied mechanical loading. During the whole process, the highest amplitude for the $\mathrm{AE}$ signals was less than $85 \mathrm{~dB}$ and the corresponding $\mathrm{AE}$ energy was relatively low as well. As discussed above, it was feasible to conclude that during the application of mechanical loading up to $2000 \mathrm{~N} \mathrm{~m}$, the main damage forms could be determined as the matrix cracking accompanying with the interface debonding. According to distribution density of $\mathrm{AE}$ signals, the quantity of these damages was much less. And what's more, due to the lack of the signals with amplitude higher than $85 \mathrm{~dB}$, so there was no fracture of reinforced fiber during the process of the application of mechanical loading. That is to say that the applied mechanical loading was insufficient to result in the fracture of reinforced fiber, which was the most severe damage mode for the composites. During the time of 168-192 s, many signals with the amplitude in the range $45-85 \mathrm{~dB}$ were exhibited. It was suggested that interface debonding was never isolated as an individual damage mechanism, always being accompanying with the matrix cracking mechanism. So during the process of single mechanical loading, the damage modes were mainly focused on the matrix cracking and debonding of interface. Meanwhile, the degree of damage was relatively slight, because there was no occurrence of the fiber fracture.

Compared with Fig. 2, as shown in Fig. 3, much denser $\mathrm{AE}$ signals were detected during the single heating process, which suggested that more quantity of $\mathrm{AE}$ events, i.e., damage or failure occurred. Among them, during the 121to 164-s phase, occasionally some high-energy AE signals with amplitude more than $85 \mathrm{~dB}$ were detected, which suggested that the fiber fracture mechanism occurred. This was attributed to thermal stress due to mismatch of the coefficient of thermal expansion (CTE) between the reinforced $\mathrm{C}$ fiber and the $\mathrm{SiC}$ matrix (the CTE of $\mathrm{SiC}$ is $4.7 \times 10^{-6} / \mathrm{K}$, the $\mathrm{CTE}$ along axial direction for $\mathrm{C}$ fiber is from $-0.72 \times 10^{-6} / \mathrm{K}$ to $-0.9 \times 10^{-6} / \mathrm{K}$, and the CTE along radial direction for $\mathrm{C}$ fiber is $\left.(22-32) \times 10^{-6} / \mathrm{K}\right)$. Meanwhile, the occurrence of a great number of AE signals with amplitude less than $80 \mathrm{~dB}$ revealed that the quantity of other failure modes, such as the matrix cracking and debonding of interface between the fiber and matrix, was relatively higher. So the degree of damage and failure was more severe during the heating process than that during the single application of mechanical loading.

Compared with Fig. 3, a great amount of AE signals were detected during the process of the combination of mechanical loading and heating, as shown in Fig. 4. The number of $\mathrm{AE}$ events reflects the degree of damage activities in materials, so a great number of damage and failure forms were produced during thus process. And what's more, compared with Fig. 3, although the distribution and shape of the $\mathrm{AE}$ characteristic signals detected were very similar, it can be seen that the various AE signals in Fig. 4 cannot be obtained by the simple superposition of the corresponding AE signals in Figs. 2 and 3. Otherwise, the number, the distribution density and the intensity of $\mathrm{AE}$ signals in Fig. 4 significantly exceed the simple superposition of corresponding AE signals from Figs. 2 and 3. So it was feasible to conclude that a synthetic effect of damage between the mechanical loading and the heating existed. Obviously, the quantity of AE signals with the amplitude more than $85 \mathrm{~dB}$ was much more, so the damage and failure of the component was more severe. It was concluded that the mutual reaction between the heating and 
mechanical loading will further deteriorate the comprehensive properties of the $\mathrm{C} / \mathrm{SiC}$ composites.

\section{Conclusions}

1. During the process of the single mechanical loading, the highest amplitude of the AE signal was less than $85 \mathrm{~dB}$, and the main damage mechanism was matrix cracking and interface debonding between the reinforced fibers and matrix, but the failure form of fiber fracture was exclusive.

2. During the single heating process, much stronger and denser AE signals were detected, which was caused by thermal stress. Occasionally, some high-energy AE signals with amplitude more than $85 \mathrm{~dB}$ were detected, which suggested that the fiber fracture mechanism occurred.

3. During the process of combination of the heating and mechanical loading, a great number of damage and failure forms including the matrix cracking, interface damage and fiber fracture were produced. From the characteristics of AE signal, it was concluded that the degree of damage during the combination of the heating and mechanical loading was much severer than the simple superposition of the corresponding $\mathrm{AE}$ signals obtained during individual ones.

Acknowledgements This work was supported by the National Scientific Instrument and Equipment Development Project under Grant No.2011YQ14014504.

\section{References}

[1] I.M. De Rosa, C. Santulli, F. Sarasini, Compos. Pt. A Appl. Sci. Manuf. 40, 1456 (2009)

[2] F. Dahmene, S. Yaacoubi, M.E.L. Mountassir, Phys. Proced. 70, 99 (2015)

[3] M.G. Droubi, R.L. Reuben, G. White, Mech. Syst. Signal Proc. 62-63, 415 (2015)

[4] P. Datt, J.C. Kapil, A. Kumar, Cold Reg. Sci. Technol. 119, 170 (2015)

[5] Y. You, Q. Teng, Z. Zhang, Q. Zhong, Mater. Sci. Eng. A 655, 277 (2016)

[6] A.A. Shah, Y. Ribakov, Mater. Des. 31, 3777 (2010)

[7] H. Suzuky, M. Takemoto, K. Ono, J. Acoust. Emiss. 11, 117 (1993)

[8] M. Suzuki, H. Nakanishi, M. Iwamoto, E. Jinen, Application of static fracture mechanisms to fatigue fracture behavior of class A-SMC composite, In: 4th Japan-US Conference on Composite Materials, (Washington, USA, 27-29 1988), paper no. A9018369 (AH), pp. 297-306

[9] P.J. De Groot, P.A.M. Wijnen, R.B.F. Janssen, Compos. Sci. Technol. 55, 405 (1995)

[10] A. Okada, T. Yasujima, T. Tazawa, Trans. Jpn. Inst. Met. 28, 1004 (1987)

[11] K.S. Suh, C.R. Lee, K.H. Park, J.W. Park, Polymer (Korea) 16, 15 (1991)

[12] S. Barre, M.L. Benzeggagh, Compos. Sci. Technol. 52, 369 (1994)

[13] J.M. Berthelot, J. Rhazi, Compos. Sci. Technol. 37, 411 (1990)

[14] M. Faudree, E. Baer, A. Hiltner, J. Collister, J. Compos. Mater. 22, 1170 (1988)

[15] N. Sato, T. Kurachi, S. Sato, O. Kamigato, J. Mater. Sci. 19, 1145 (1984)

[16] J.M. Berthelot, J. Reinf. Plast. Comp. 7, 284 (1988)

[17] I. Narisawa, H. Oba, J. Mater. Sci. 19, 1777 (1984)

[18] I. Narisawa, H. Oba, J. Mater. Sci. 20, 4527 (1985)

[19] M. Giordano, A. CalabroÁ, C. Esposito, A. D’Amorec, L. Nicolai, Compos. Sci. Technol. 58, 1923 (1998) 